\title{
A Novel Machine Learning-based Energy Consumption Model of Wastewater Treatment Plants
}

\author{
Shike Zhang ${ }^{1}$, Hongtao Wang ${ }^{1,2 *}$, Arturo A. Keller ${ }^{3}$
}

1 College of Environmental Science and Engineering, Key Laboratory of Yangtze River Water Environment, Ministry of Education, UNEP-Tongji Institute of Environment for Sustainable Development, Tongji University, 1239 Siping Rd, Shanghai, 200092, China

2 Shanghai Institute of Pollution Control and Ecological Security, Tongji University, 1239 Siping Rd, Shanghai, 200092, China.

3 Bren School of Environmental Science and Management, UC Santa Barbara, Santa Barbara, California 93106, United States

*Corresponding author: Email: hongtao@tongji.edu.cn (Hongtao Wang)

\section{Detailed steps of random forest algorithm (explanation to Fig.2):}

First, the bootstrapping method was used to randomly obtain $\mathrm{N}$ samples $\left(\mathrm{S}_{1}, \mathrm{~S}_{2} \ldots \ldots \mathrm{S}_{\mathrm{n}}\right)$ from the total sample $\mathrm{S}$. A decision tree was trained using $\mathrm{n}$ sets of samples, and the above process was repeated $\mathrm{m}$ times. Subsequently, a random forest comprising $\mathrm{m}$ decision tree models was obtained. At this time, the target data were entered into each decision tree model, and independent category outputs $\mathrm{K}_{1}, \mathrm{~K}_{2} \ldots \ldots \mathrm{K}_{\mathrm{m}}$ from each decision tree model was generated. By computing the votes, each of which represents the category output of a decision, the final results of the classification or regression of the target data were obtained. Because the random forest is composed of multiple decision trees, the data need not be divided into training and test sets (Breiman 2001). Theoretically, the accuracy of this model can be calculated from the voting results of a single decision tree model. Sample $\mathrm{S}$ is the data of this study.

Table S1 Algorithm Principle

\begin{tabular}{cc}
\hline Algorithm & Principle \\
\hline Random Forest (RF) & Bagging and Decision Tree \\
Boosting Tree & AdaBoost and Decision Tree \\
Gradient Boosting Decision Tree (GBDT) & Gradient Boosting and Decision Tree \\
XGBoost & Improved GBDT Algorithm \\
\hline
\end{tabular}




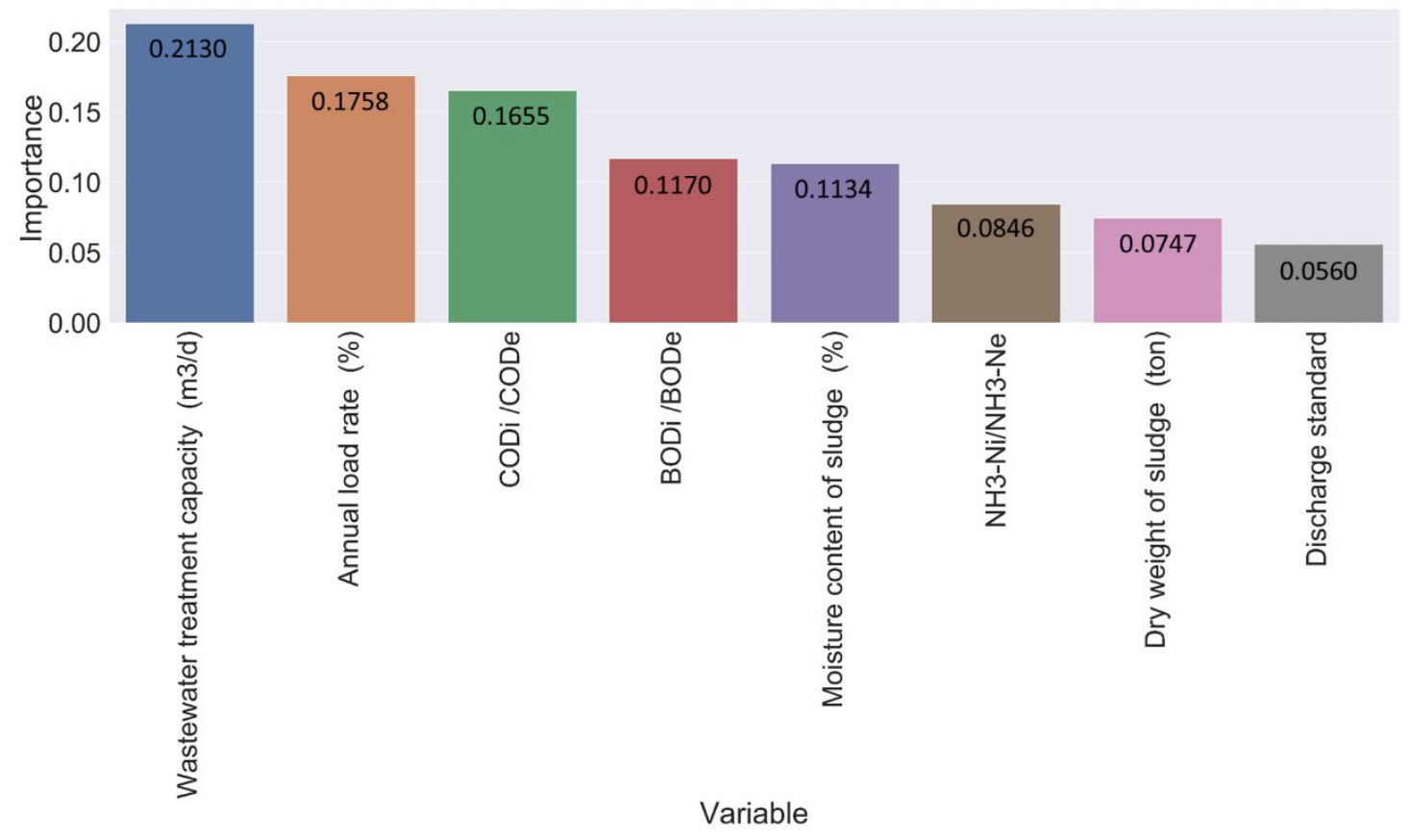

Fig. S1 Variables and their importance
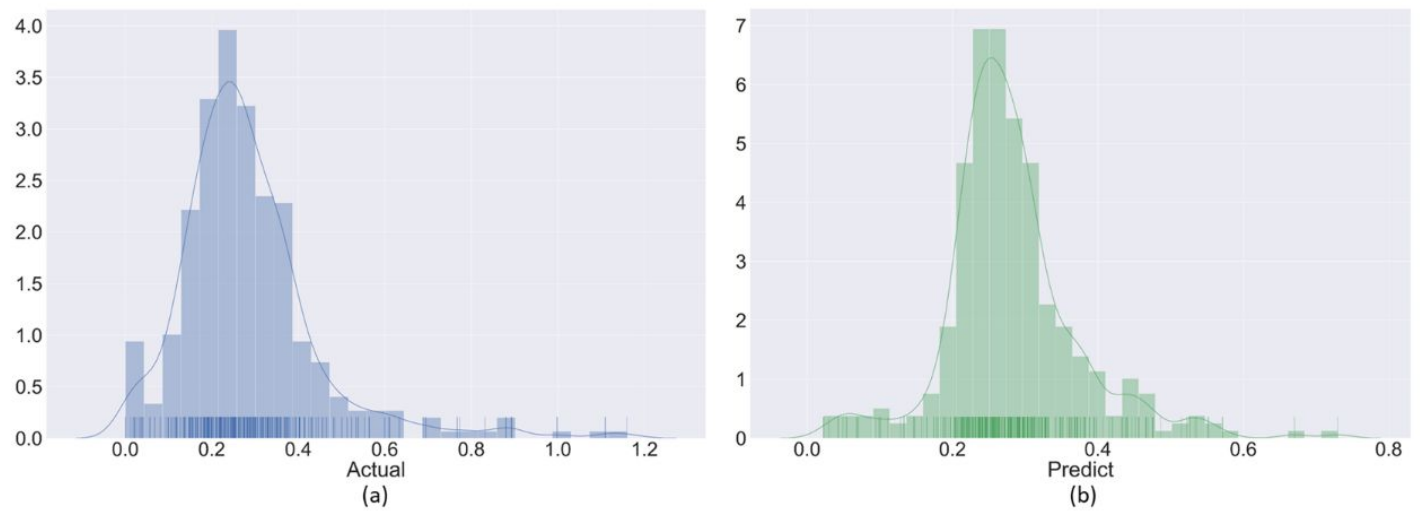

Fig. S2 Detailed Kernel frequency distribution representing (a) Actual and (b) Predicted unit electricity consumption for 347 WWTPs.

\section{Biological processes of WWTPs in China:}

There are a lot of biological wastewater treatment technologies are used in China. The predominant ones include anaerobic-anoxic-oxic (A2/O), oxidation ditch (OD), sequencing batch reactor (SBR), anoxic-oxic (A/O), membrane bio-reactor (MBR), BIOLAK, biofilter, wetland, oxidation pond, biological contact oxidation, activated sludge, and fluidized bed etc. Among them, OD is the most commonly used technology and about $32.8 \%$ of WWTPs adopts this technology. The second most widely used technology is A2/O with 32.5\% of WWTPs adopting this technology. The third most popular technology is SBR with an adoption rate of 16.5\%. The fourth most popular technology is $\mathrm{A} / \mathrm{O}$ whose adoption rate is $16.5 \%{ }^{1}$.

\section{Reference}

1 Luo, L., M. Dzakpasu, B. Yang, W. Zhang, Y. Yang and X. C. Wang (2019). "A novel index of total oxygen demand for the comprehensive evaluation of energy consumption for urban wastewater treatment." Applied Energy 236: 253-261. 